\title{
Forecasting of COVID 19 Cases in Karnataka State using Artificial Neural Network (ANN)
}

\author{
Rashmi P. Shetty $^{1}$ (1) P. Srinivasa Pai ${ }^{1}$
}

Received: 15 August 2020/ Accepted: 17 May 2021/Published online: 18 June 2021

(C) The Institution of Engineers (India) 2021

\begin{abstract}
COVID-19 is a pandemic that has caused lot of deaths and infections in the last 2 months and is showing an increasing trend not only in the number of infections and deaths, but also in the recovery rate. Accurate prediction models are very much essential to make proper forecasts and take necessary actions. This study demonstrates the capability of Multilayer Perceptron (MLP), an Artificial Neural network (ANN) model for forecasting the number of infected cases in the state of Karnataka in India. It is trained using a fast training algorithm namely, Extreme Learning machine to reduce the training time required. The parameters required for the forecasting model have been selected using partial autocorrelation function, which is a conventional method, and its performance has been compared with parameters selected using cuckoo search (CS) algorithm, which is a very popular metaheuristic optimization algorithm. The testing of the forecasting model has been done, and comparison between the two parameter selection methods as well as with MLP with conventional backpropagation has been carried out. Use of CS algorithm has resulted in a better forecasting performance based on mean absolute percentage error (MAPE), with a value of $6.62 \%$ on training data and $7.03 \%$ on the test data. Further to check the efficacy of the model, the data of COVID-19 cases of Hungary from 4 March to 19 April 2020 have been used, which resulted in a MAPE of $1.55 \%$, thereby establishing the robustness of the proposed ANN model for forecasting COVID-19 cases for the state of Karnataka.
\end{abstract}

Rashmi P. Shetty

iprashmi@nitte.edu.in

1 Dept. of Mechanical Engineering, NMAM Institute of Technology, Nitte, Karkala, Karnataka, India
Keywords Forecasting - Artificial neural network · Multilayer perceptron - Partial autocorrelation function . Mean absolute percentage error $\cdot$ Cuckoo search

\section{Introduction}

Severe acute respiratory syndrome (SARS) corona virus 2 is a virus strain that is causing severe respiratory disease, which is popularly known as COVID-19. World Health Organization (WHO) has declared it as a pandemic as on 11-03-2020. As on 04-05-2021, 154 million confirmed cases have been reported by the Johns Hopkins University [1]. In India, as on 4-05-2021, 20.7 million cases have been reported with 226,000 deaths [2]. This has disturbed the normal life of ordinary people and has affected many both economically and from other aspects. The lockdown imposed by the government in the initial stages has affected the livelihood of millions of people and has significant repercussions. There has been lot of stress on the administration for managing the spread of this virus, and accordingly there is a need for forecasting the probable number of cases, so that the infections can be managed effectively without undue strain on the health infrastructural facilities of the country.

There have been several efforts regarding use of mathematical models and artificial intelligence (AI) tools in modeling, prediction and forecasting of the infected cases, recovered cases, mortality rate, etc., in the last several months. It is very important to model the spread and effect of such diseases, particularly creating a pandemic. AI modeling techniques are able to generate high-quality predictive models and can help in the various aspects of disease management. The data are readily available, and they are valid and from reliable sources. There is a need for 
a mechanism to manage the data efficiently and AI and in particular machine learning models can help in doing the same [3].

This paper presents a forecasting model based on multilayer perceptron, a popular, supervised and feed forward neural network model. The parameter selection for the model is based on PACF and using Cuckoo Search algorithm. The model has been checked for its efficiency, based on the data pertaining to Hungary.

\section{Related Work}

There has been a lot of work reported about the use of Artificial Intelligence and Machine learning (ML) for studying the various aspects of COVID-19. To review a few literatures, Shinde et al. [4] presented an overall, comprehensive analysis of different forecasting models for COVID-19 to help the organizations. The aspects covered include forecasting, impacts and control measures. The models have been studied from the point of view of challenges in using these and recommendations. The forecasting methods have been grouped into four namely big data sets accessed from WHO and national data sources, social media data, stochastic models and use of machine learning techniques. They recommend the need to test the models globally for better global forecasting.

Swapnarekha et al. [5] presented a neural network approach in the prediction, diagnosis of COVID-19 for its effective control. The performance analysis of Deep learning, ML and statistical approaches has been performed which helps researchers in developing better models for predicting the disease. Pinter et al. [6] proposed an alternative to the susceptible-infected-resistant (SIR)-based models by proposing hybrid learning methods which use multi-layered perceptron with imperialist-competitive algorithm (MLP-ICA) and adaptive network-based fuzzy inference system (ANFIS). These models have potential, when compared to epidemiological models and MLP-ICA performed better than ANFIS and gave good results on validation data.

Rustam et al. [7] studied different models based on ML to predict the patient numbers affected by the disease and included least absolute shrinkage and selection operator (LASSO), linear regression (LR), exponential smoothing (ES) and support vector machine (SVM). Different predictions such as, number of newly infected cases, the number of deaths and number of recoveries in the future ten days were made. ES performs well in the current forecasting domain based on the nature and size of the data. LR and LASSO perform well for forecasting death rate and confirmed cases. Sujath et al. [3] presented a model to predict COVID-19 spreading. The models studied include
LR, MLP and Vector autoregression (VAR) method using Kaggle data to forecast the epidemiological example of the disease and number of cases in India. It was found that, MLP performed better than LR and VAR using Weka an Orange by considering the data from John Hopkins University.

Saba and Elsheikh [8] studied statistical-based method namely nonlinear autoregressive artificial neural networks (NARANN) and autoregressive integrated moving average (ARIMA) for modeling and forecasting the prevalence of this disease in Egypt. The data of confirmed cases are used to train the proposed models. NARANN performed better than ARIMA and found to be useful for multi-step forecasting with high coefficient of determination, and the forecasted error was less than 5\%. Hassan [9] used realtime data to investigate a hybrid model namely ensemble empirical mode decomposition (EEMD)-ANN, to predict the daily trend of COVID-19. The EEMD method is used for data denoising, and ANN is used to build the model. The model was compared with statistical techniques like regression analysis and moving average. The proposed model has very low mean squared error (MSE) and high $\mathrm{R}^{2}$ values. Car et al. [10] used a data set of 406 locations for a time duration of 51 days, which was converted into a regression data set and used to train a MLP model. From among the three categories of models, the deceased patient model was highly robust. There was good robustness for the infected patient model and poor performance for the recovered patient model. Tamang et al. [11] used ANN curve fitting techniques in predicting and forecasting the confirmed and death cases of COVID-19 in India, USA, France and UK using the data from South Korea and China. The results indicate that ANN is most suitable for COVID19 forecasting of any nation. Mollalo et al.[12] made a review of countrywide modeling of COVID-19 cases in USA, by using ML techniques and identified 57 variables for checking the performance of MLP to predict the cumulative COVID-19 occurrence rates in continental USA. Proposed model was able to establish $65 \%$ correlation with the ground truth for the hold out samples.

This review highlights the wide range of models and areas covered, with scope for use of ML-related methods for modeling, prediction and forecasting of the disease.

\section{Multilayer Perceptron-based Neural Network Model}

ANN due to its ability to map meaningful relationships in the data is becoming popular day by day in medical field for clinical diagnosis, drug development, improved gene editing and in prediction and forecasting of diseases. Feed forward neural networks are the simplest but powerful class 
of neural networks which find wide application due to their ability to model any nonlinear relation underlying the data, which otherwise are difficult to be handled by conventional techniques.

The main feature of ANN, which makes its application universal, is its learning ability and thereby adjusts synaptic weights and biases to best fit the given environment. Back Propagation (BP) algorithm, though very popular and used in many areas, suffers from many problems. This iterative algorithm, in addition to being slow and time consuming, suffers from local minima problem. There are most chances of overtraining of the network, if the appropriate stopping criteria are not set during learning. Use of many simulation parameters makes it sensitive to parameter tuning and needs more human intervention [13]. Extreme learning machine (ELM) is a powerful technique presented by $\mathrm{G}$. B Huang [14], which overcomes the disadvantages of BP. Good generalization performance with less simulation parameters are the added advantages of ELM in training MLP, when compared to use of BP in training MLP as well as support vector machines (SVM) [15].

\section{Methodology}

\section{Multilayer Perceptron Neural Network}

Multilayer perceptron is a popular feed forward model, which uses Backpropagation (BP) algorithm. It consists of one or more hidden layer with nonlinear sigmoidal activation function as given in Eq. (1)

$V_{j}^{\mu}=\frac{1}{1+e^{\sum w_{\mathrm{ji}} x_{i}^{\mu}+b_{\mathrm{ji}}}}$

where, $x_{i}^{\mu}$ is the input data with $i=1,2, \ldots . . s$ input features and $\mu=1,2, \ldots n$ input patterns. $\mathrm{b}_{j}$ and $w_{\mathrm{ji}}$ are the bias and weights, respectively, between hidden and input layer with $j=1,2,3 \ldots p$ is the neurons in the hidden layer. MLP possesses high degree of connectivity and constructs global approximation. Hence, it is capable of mapping any nonlinear relationships [13].

\section{Extreme Learning Machine (ELM) Algorithm}

This algorithm, being a simple and non-iterative method, draws the attention of researchers from various fields. The one pass learning helps in saving the learning time considerably and thus finds a major application in emerging areas such as pattern recognition, image analysis, bioinformatics and other prediction and forecasting purposes involving big data [14].

The ELM algorithm is as follows:
For $N$ arbitrary distinct training data $\left(x_{i}, t_{i}\right)$, where $X_{i} \in$ $R^{n}$ is $n * 1$ input and $t_{i} \in R^{m}$ is $m * 1$, target vector and Q hidden nodes, if the activation function $g(x)$ is used, then the output $O_{j}$ of the model can be expressed mathematically as:

$O_{j}=\sum_{i=1}^{Q} \beta_{i} g_{i}\left(w_{i} x_{j}+b_{i}\right), \quad j=1, \ldots ., N$

where, $w_{i}$ and $\beta_{i}$ with $i=1,2, \ldots Q$ are the weight matrix between hidden, input layers and between output, hidden layers of size $n^{*} Q$ and $Q^{*} \mathrm{~m}$, respectively. $b_{i}$ is the bias of $i$ th hidden node. Equation (2) can be expressed as

$H \beta=T$

where,

$H\left(w_{1}, \ldots w_{N}, b_{1}, \ldots b_{N}, x_{1}, \ldots \ldots x_{N}\right)$
$=\left[\begin{array}{ccc}g\left(w_{1} x_{1}+b_{1}\right) & \ldots & g\left(w_{Q} x_{1}+b_{Q}\right) \\ \cdot & & \cdot \\ \cdot & \ldots & \cdot \\ \cdot & & \cdot \\ g\left(w_{1} x_{N}+b_{1}\right) & \ldots & g\left(w_{Q} x_{N}+b_{Q}\right)\end{array}\right]_{N * Q}$

$\beta=\left[\begin{array}{c}\beta_{1}^{T} \\ \cdot \\ \cdot \\ \cdot \\ \beta_{Q}^{T}\end{array}\right]_{Q * m}$

and

$T=\left[\begin{array}{c}t_{1}^{T} \\ \cdot \\ \cdot \\ \cdot \\ t_{N}^{T}\end{array}\right]_{N * m}$

$\beta=H^{\dagger} T$

The output weight matrix is found using Eq. 7 where $H^{\dagger}$ is the Moorse-Penrose generalized inverse of matrix $H$ [14].

\section{Cuckoo Search (CS) Algorithm}

The metaheuristic algorithms are inspired by natural processes and are stochastic in nature. In 2009, Yang and Deb introduced CS algorithm, which is based on cuckoo bird's obligate brood parasitic nature and Levy flight behavior of animals. This algorithm is fast and efficient in exploring the optimal solution, has less chances of being trapped in local minima, because of the Levy flight mechanism to generate new solutions, and is a popular nature inspired optimization algorithm [16-18]. 


\section{PACF}

Partial autocorrelation function is a method used to perform time series data analysis. It is used to evaluate the order of the autoregressive model. It is mainly useful in determining the lag in autoregressive (AR) and ARIMA models. It is helpful in understanding the linear dependence of a value in the time series data with its lagged value [19].

\section{Model Development}

Three MLP-based models are trained with ELM learning algorithm by using CS and PACF for selection of input parameters. Details of the models developed are presented in Table 1. When sigmoidal activation function is used in the ELM based model, there are no simulation parameters to be tuned. Figure 1 provides proposed model (Model-2) framework. The simulation parameters of Model-3 namely learning rate, momentum rate have been set to be 0.15 , 0.08 , and the stopping criteria have been set as maximum of 1000 epochs or minimum error of $1 \times 10^{-3}$.

\section{Data Collection}

Data of COVID-19 infected cases in Karnataka state of India are provided by the government and are available in https://www.google.com/search?client=firefox-b-d\&q=covid $+19+$ tracker. Figure 2 presents the daily reported cases of COVID-19 in Karnataka from 10 March 2020 to 21 July 2020, which shows a steep increase after 10th June. The data have been normalized to bring it in $0-1$ range by using Eq. (8). This helps in providing uniform contribution of each data in model building.

$X^{\prime}=\frac{X}{X_{\max }}$

where $X^{\prime}$ is the data after normalization, $X$ is the actual data and $X_{\max }$ is the maximum value in the dataset. The simulation of the present work has been done in MATLAB R2014a on a personal computer with an Intel i5-6200U $2.3 \mathrm{GHz} \mathrm{CPU}$ and $4 \mathrm{~GB}$ RAM.

\section{Selection of Input Parameters}

Once the optimum number of input parameters $(n)$ is selected, the data set is prepared as shown in Fig. 3, from total of $N$ number of data instances. In the present work, the total number of data instances is 133 . The objective function for CS algorithm has been considered as; minimize the mean absolute percentage error (MAPE) between actual and predicted values as given in Eq. (9). CS algorithm selected the optimum number of input parameters as 5 . The MAPE (\%) variation with number of iterations of CS is presented in Fig. 4. The partial correlogram of PACF method for the data is presented in Fig. 5. It can be analyzed from the figure that only one input parameter is having high correlation with more than $95 \%$ significance level. Hence, 128 and 132 datasets from 133 total data instances are generated with input and output as detailed in Table 1 for CS-based and PACF-based parameter selection, respectively. Out of which $85 \%$ is used for training the ANN model and rest for the testing the same. The selection of the data set has been done randomly, to take care of the generalization performance and avoiding overfitting.

$\operatorname{MAPE}(\%)=\frac{1}{N} \sum_{i=1}^{N} \frac{\left|y_{p}(i)-y_{a}(i)\right|}{y_{a}(i)} \times 100$

$\operatorname{RMSE}=\sqrt{\frac{1}{N} \sum_{i=1}^{N}\left(y_{p}(i)-y_{a}(i)\right)^{2}}$

where $y_{p}(i), y_{a}(i)$ are predicted and actual values of number of COVID-19 cases at $i$ th time instance and $N$ represents the total number of datapoints considered. The values of the parameters set for CS algorithm in this work have been provided below.

(1) Probability $P_{a}=0.25$.

(2) Stopping criteria $=100$ iterations.

(3) Population size $n=10$.

(4) Distribution factor $\beta=1.5$. [20]

Table 1 Model details

\begin{tabular}{lllll}
\hline Models & Input parameter selection & Input & Output & Learning algorithm \\
\hline Model-1 & PACF & Xt-1 & Xt & ELM \\
Model-2 & CS & Xt-5, Xt-4, Xt-3, Xt-2, Xt-1 & Xt & ELM \\
Model-3 & CS & Xt-5, Xt-4, Xt-3, Xt-2, Xt-1 & Xt & BP \\
\hline
\end{tabular}



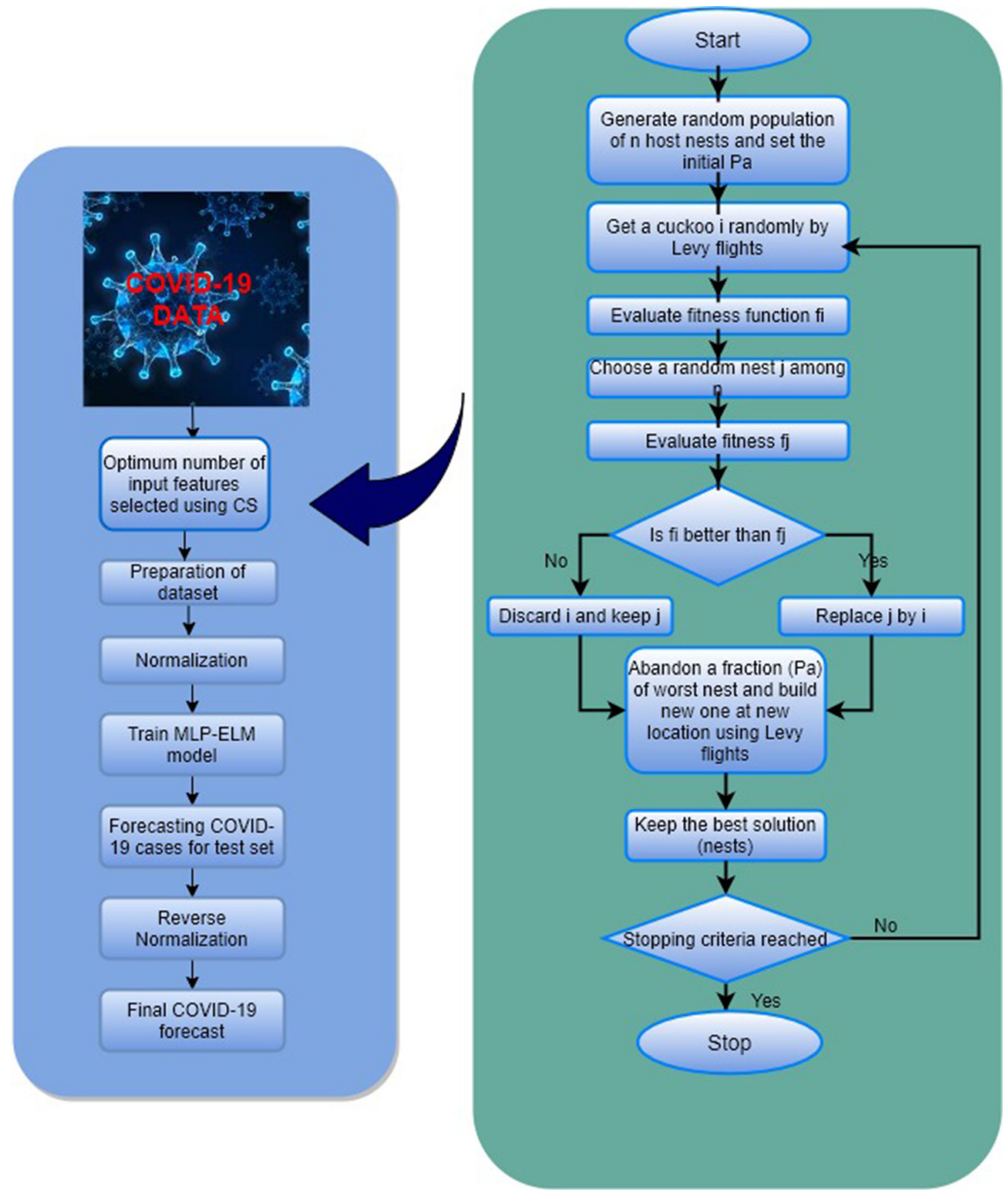

Fig. 1 Proposed COVID-19 forecasting model framework

\section{Results and Discussion}

To estimate the performance of the models, mean absolute percentage error (MAPE) and root mean square (RMSE) as given in Eq. (9) and (10), respectively, have been used as the performance metrics.

\section{Model-1}

The hidden neuron numbers have been varied to obtain better accuracy. The performance of Model- 1 for different hidden neurons is shown in Table 2. It can be seen that the model gives minimum MAPE (\%) of 20.73 for 25 hidden neurons on test data. Hence, the optimum model configuration is 1:25:1. The actual and Model-1 predicted values are shown in Fig. 6. 
Fig. 2 Statistics of number of active COVID-19 cases in Karnataka from 10/03/2020 to 21/07/ 2020

Fig. 3 Dataset preparation

\section{Model-2}

Performance of Model-2 for varied number of hidden neurons is shown in Table 3 . It is noted that when the number of neurons is 10 , the model showed best performance with least MAPE (\%) value of 7.03 on test data. It is also observed that further increase in number of hidden neurons leads to decaying model accuracy. Hence, the optimal model configuration is found to be 5:10:1. Figure 7 shows actual and Model-2 forecasted values for the test set considering 1-step, 3-step and 5-steps.
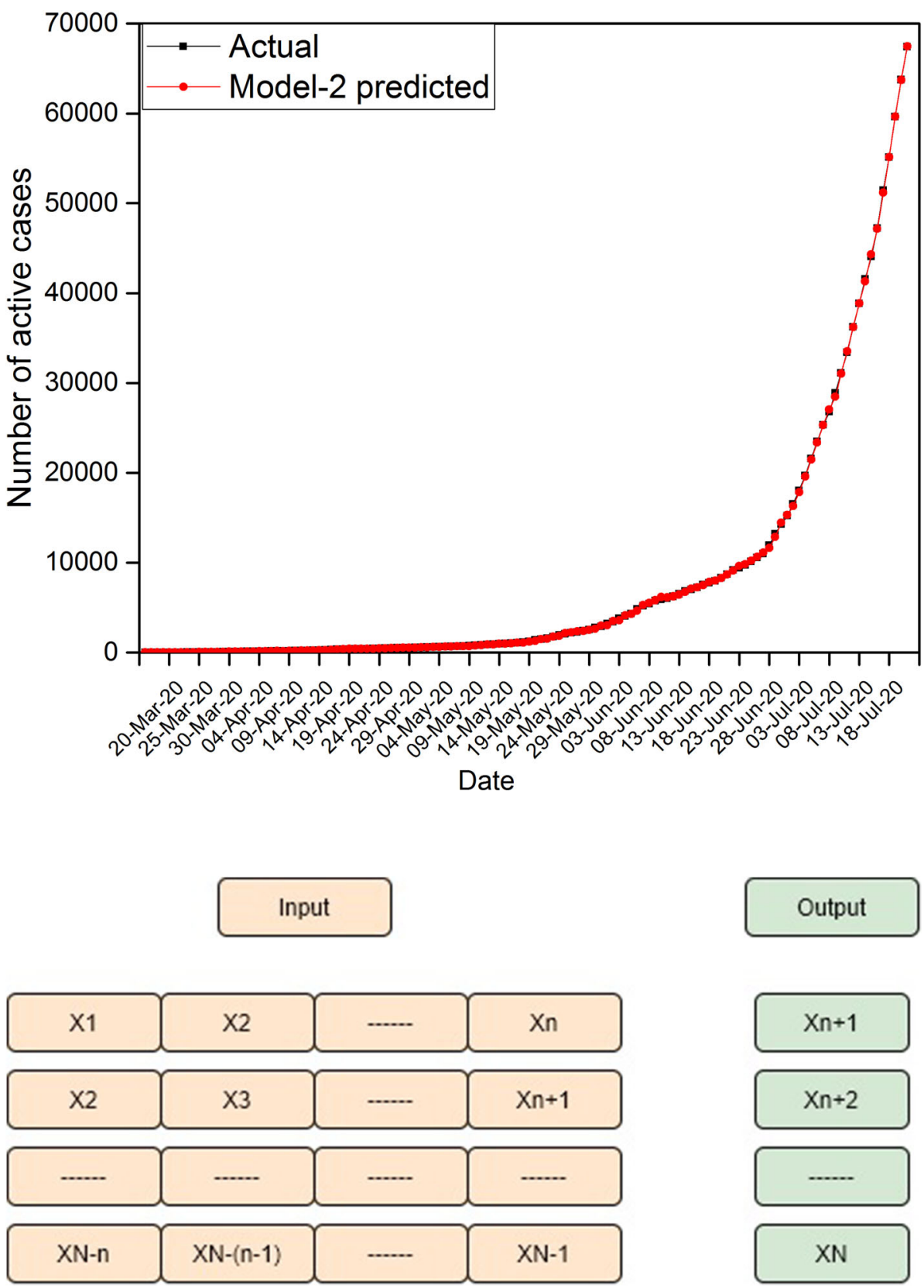

Input

$x n+1$

$\mathrm{X} n+2$

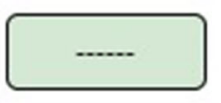

$\mathrm{XN}$ 


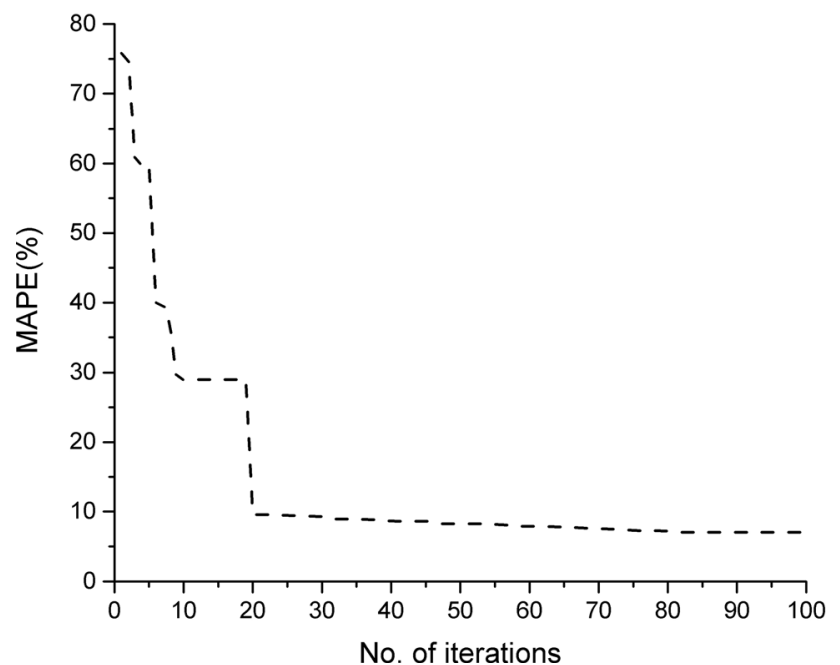

Fig. 4 Convergence curve of CS algorithm

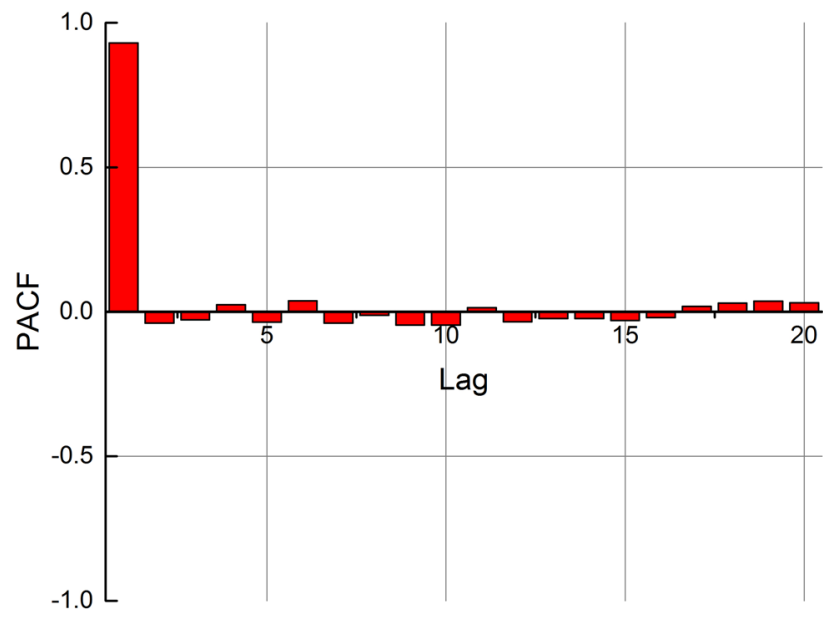

Fig. 5 Partial correlogram of COVID-19 data

training MAPE (\%). Hence, it takes more computation time in comparison with Model-1. However, it is worth noting that proper selection of input parameters has a considerable effect on model performance, thus resulting in lower
MAPE (\%) value of 7.03 by Model-2 in contrast to 20.73 of Model-1 on test data. Graphical illustration of Model-2 predicted and actual COVID-19 infected cases is presented in Fig. 2. A good agreement of predicted values with actual is found. Thus, the CS algorithm, which is used in a wide variety of applications, is proved successful in improving the forecasting accuracy of the ANN model, when used with optimal input parameter selection.

For proving the novelty of the present work and to understand the benefits of using the present model using ELM algorithm, it has been compared with MLP neural network model based on BP algorithm, which is a widely used popular technique. The details of Model-3 are provided in Sect. 4.5. CS-based feature selection is used in both models for comparison. From Table 5, it can be observed that, Model-2 gave accurate results with MAPE (\%) of 7.03 on test data in comparison with 21.23 of Model-3. Model-2 being ELM-based model takes less training time of $0.04910 \mathrm{~s}$ in contrast to $0.9981 \mathrm{~s}$ taken by Model-3. Further, Model-2 resulted in comparatively compact network structure taking only 10 hidden neurons. The results show a clear advantage of the proposed model with respect to accuracy, computation speed, model structure and hence the novelty.

\section{Multistep Forecasting}

To prove the suitability of the proposed model in predicting the COVID-19 cases for future time horizon, a multistep forecasting is performed and results are presented for 3 -step and 5-step ahead forecasting. Each time step represents days in the present case. The multistep forecasting results presented in Table 6 and Fig. 7 show that the model proposed is capable of predicting for multiple future time steps with reasonable accuracy. It can be observed that, with the increase in time step the error increases. This is obvious because of the accumulation of the error of the previous time step, which is fed as the input to the next time step prediction.

Table 2 Forecasting performance of Model-1

\begin{tabular}{lcccrr}
\hline Number of hidden neurons & 10 & 15 & 20 & 25 & 30 \\
\hline MAPE (\%) on training data & 39.33 & 22.20 & 19.90 & $\mathbf{1 6 . 9 7}$ & 19.25 \\
MAPE (\%) on test data & 66.70 & 37.09 & 26.14 & $\mathbf{2 0 . 7 3}$ & 27.92 \\
RMSE on test data & 544.68 & 253.46 & 148.82 & $\mathbf{1 0 5 . 6 1}$ & $\mathbf{0 . 0 0 3 9 1 0}$ \\
CPU time (s) & 0.003127 & 0.003074 & 0.003973 & 0.004148 \\
\hline
\end{tabular}

The bold values show the optimum values with less error 
Fig. 6 Actual versus predicted number of cases from Model-1

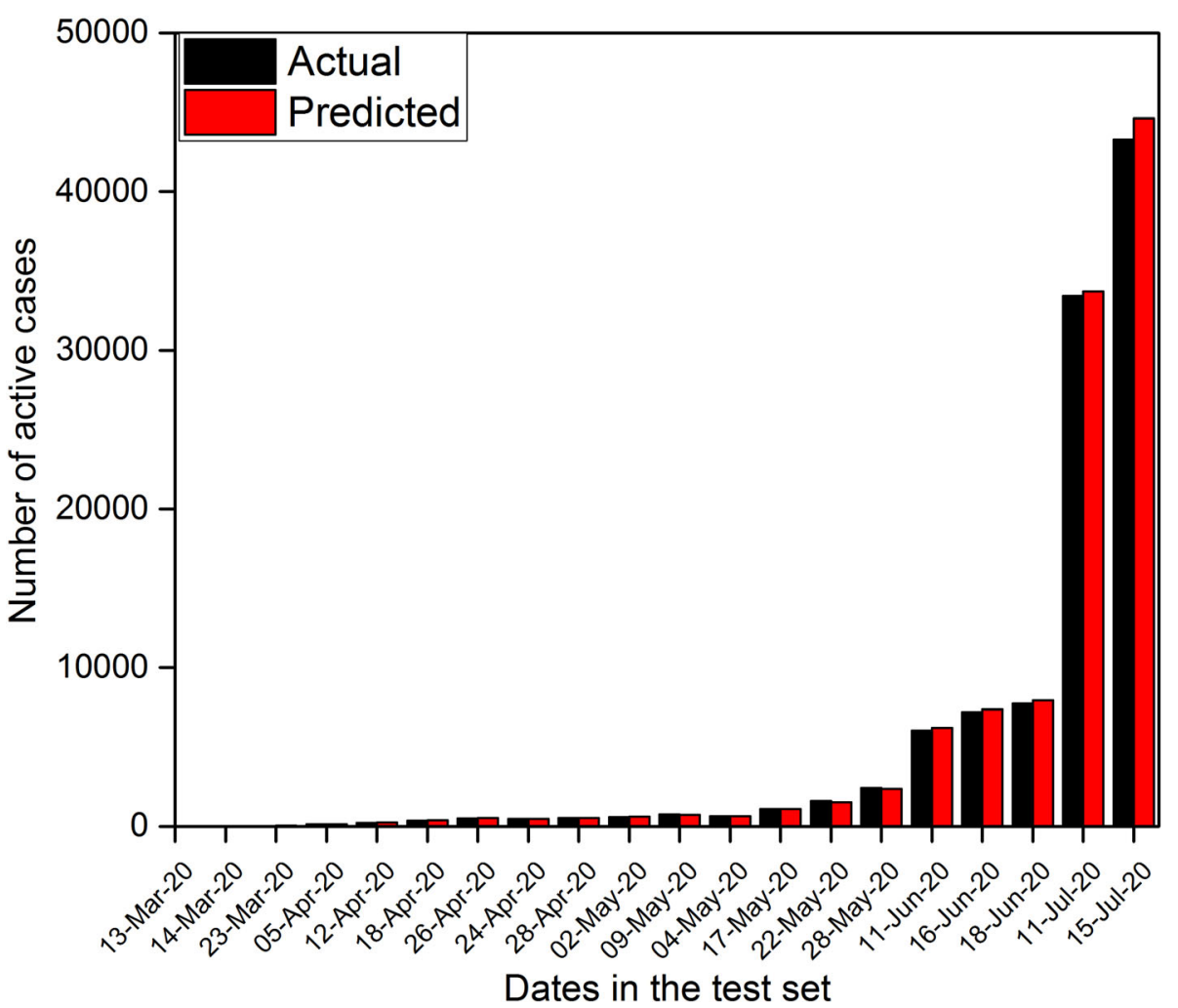

Table 3 Forecasting performance of Model-2

\begin{tabular}{|c|c|c|c|c|c|}
\hline Number of hidden neurons & 5 & 10 & 15 & 20 & 25 \\
\hline MAPE $(\%)$ on training data & 15.85 & 6.62 & 9.69 & 14.27 & 14.86 \\
\hline MAPE $(\%)$ on test data & 17.54 & 7.03 & 11.52 & 17.07 & 17.98 \\
\hline RMSE on test data & 53.86 & 28.97 & 35.77 & 45.54 & 49.31 \\
\hline CPU time (s) & 0.04551 & 0.04910 & 0.05262 & 0.05280 & 0.04551 \\
\hline
\end{tabular}

The bold values show the optimum values with less error

\section{Efficiency of the Proposed ANN Model}

To prove the effectiveness, the proposed Model-2 is applied to COVID-19 data of Hungary from 4-March 2020 to 19-April 2020 available at: https://www. worldometers.info/coronavirus/country/hungary/ and used by Gergo Pinter et al. [6]. The results obtained from the model are provided in Table 7. It can be noted from the table that least MAPE (\%) of 1.55 and 5.34 is obtained on training and test data, respectively, for 45 number of hidden neurons. The optimum number of input parameters is selected as four by the CS algorithm. The actual and forecasted values illustrated graphically in Fig. 8 show good agreement. The proposed Model-2 in this work proves to be superior to the hybrid MLP-ICA model used by [6], as Model-2 has resulted in a MAPE (\%) of 1.55 in contrast to $23.15 \%$ for MLP-ICA. Further, since Model-2 uses ELM learning algorithm, it takes only $0.05807 \mathrm{~s}$, as it works only in one epoch. However, since MLP-ICA uses an iterative learning method, it certainly consumes more computation time. Hence, Model-2, which uses ELM for learning, and CS for input parameter selection proves to be faster with better generalization performance. Further, a comparison of the proposed model with the state-of-the-art techniques found in the literature [21,22] is provided in Table 8 and the results clearly establish the effectiveness of the model. 
Fig. 7 Actual versus multistep predicted number of cases from Model-2

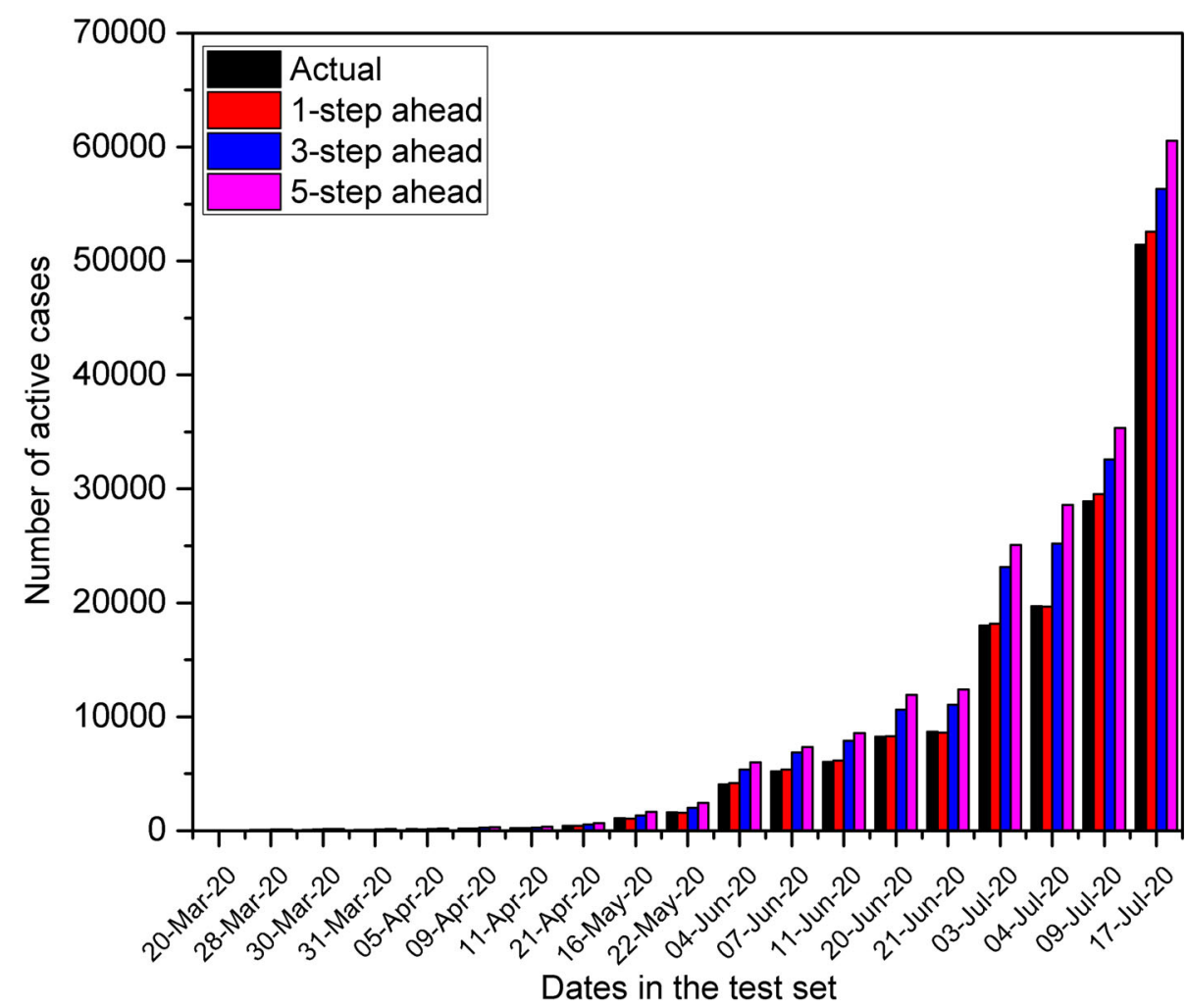

Table 4 Comparison of performances of Model-1 and Model-2

\begin{tabular}{llccc}
\hline Model & Optimal configuration & MAPE $(\%)$ on training data & MAPE $(\%)$ on test data & CPU time (s) \\
\hline Model-1 & $1-25-1$ & 16.97 & 20.73 & 0.003910 \\
Model-2 & $5-10-1$ & 6.62 & 7.03 & 0.04910 \\
\hline
\end{tabular}

Table 5 Comparison of performances of Mode-2 and Model-3

\begin{tabular}{llccc}
\hline Model & Optimal configuration & MAPE $(\%)$ on training data & MAPE $(\%)$ on test data & CPU time (s) \\
\hline Model-2 & $5-10-1$ & 6.62 & 7.03 & 0.04910 \\
Model-3 & $5-35-1$ & 20.84 & 21.23 & 0.9981 \\
\hline
\end{tabular}

Table 6 Multistep forecasting performance of Model-2

\begin{tabular}{lccc}
\hline Index & 1-step & 3-step & 5-step \\
\hline MAPE (\%) & 7.03 & 9.02 & 10.93 \\
RMSE & 28.97 & 37.17 & 45.06 \\
CPU time (s) & 0.0491 & 0.0496 & 0.0503 \\
\hline
\end{tabular}


Fig. 8 Actual versus predicted number of cases for Hungary data set from Model-2

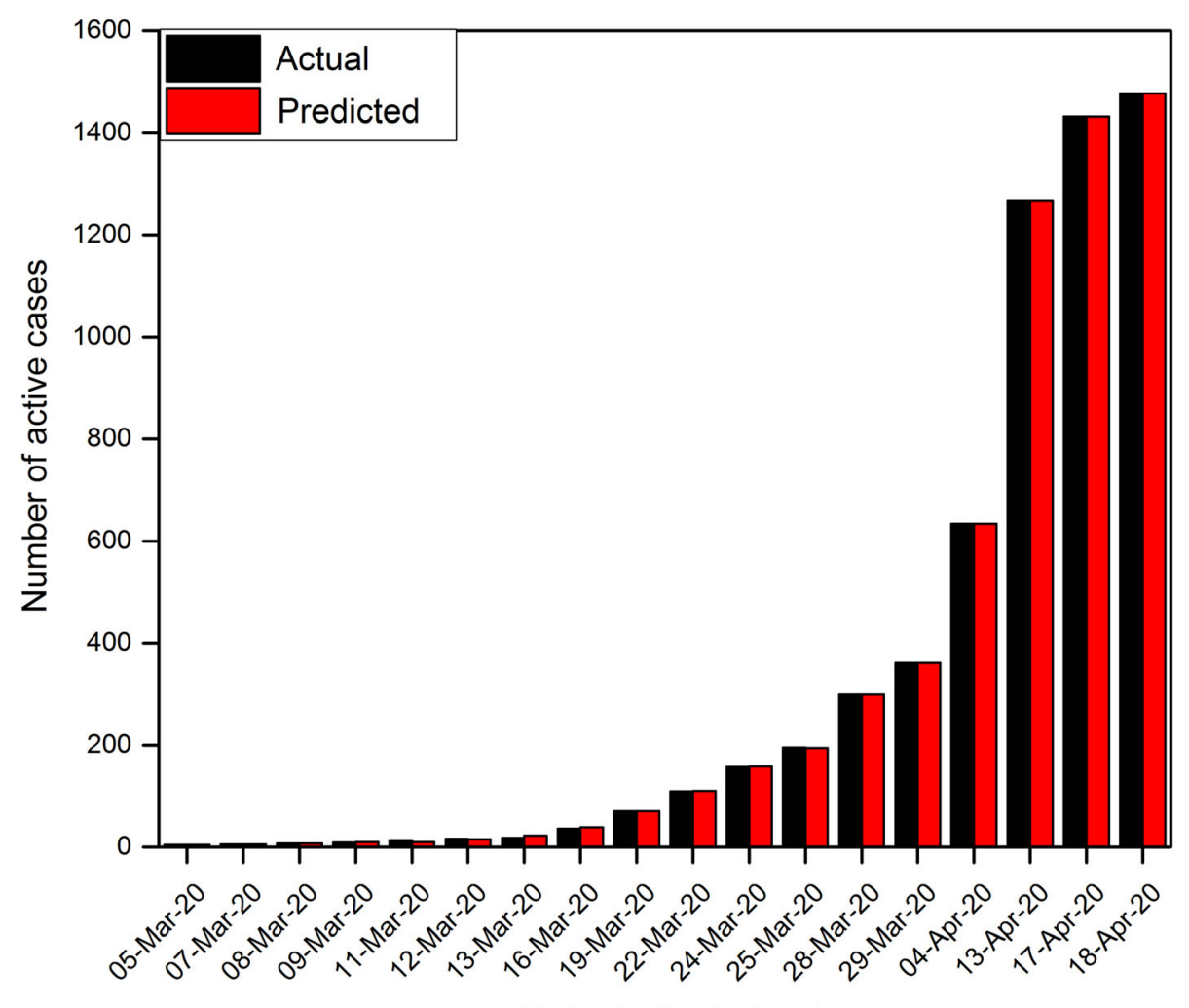

Dates in the test set

Table 7 Model-2 forecasting performance for Hungary data (Case study)

\begin{tabular}{llllll}
\hline Number of hidden neurons & 25 & 30 & 45 & 50 & 55 \\
\hline MAPE(\%) on training data & 18.31 & 4.06 & $\mathbf{1 . 5 5}$ & 2.15 & 3.65 \\
MAPE (\%) on test data & 23.58 & 9.17 & $\mathbf{5 . 3 4}$ & 5.98 & 8.95 \\
CPU time (s) & 0.05745 & 0.05906 & $\mathbf{0 . 0 5 8 0 7}$ & 0.06674 & 0.07020
\end{tabular}

The bold values show the optimum values with less error

Table 8 Comparison with state-of-the-art techniques

\begin{tabular}{llr}
\hline Technique & Region & MAPE $(\%)$ \\
\hline Proposed model & Karnataka & 7.03 \\
ANFIS [21] & Italy & 199.52 \\
Weibull model using iterative weighting [22] & India & 80.47 \\
\hline
\end{tabular}

\section{Conclusions}

Artificial neural network models based on extreme learning machine (ELM) technique are developed for forecasting COVID-19 cases in state of Karnataka, India. An effort is made to use an efficient metaheuristic algorithm namely cuckoo search (CS) for selection of input parameters for the forecasting model. The results are then compared with that of partial autocorrelation function (PACF)-based parameter selection to demonstrate the advantage of using CS in forecasting model building. To establish the effectiveness of the forecasting model, the model has been tested using COVID-19 data of Hungary and results have been compared with the other state-of-the-art techniques used for COVID-19 forecasting available in the literature. 
To check the feasibility of the model for future time horizons, multistep forecasting has been performed.

The results of this study reveal that.

(i) MLP-ELM-based forecasting model results in quick learning with less number of simulation parameters.

(ii) Use of CS optimization algorithm in Model-2 for input parameter selection results in noticeable reduction in MAPE (\%) to 7.03 in contrast to 20.73 of Model-1, which is based on PACF on test data.

(iii) The proposed forecasting model that combines ELM and CS is found to be effective, when tested using Hungary data and compared with MLP-ICA method found in [6], resulting in 1.55 MAPE (\%) against $23.15 \%$ on training data.

(iv) MAPE (\%) of 9.02 and 10.93, respectively, for 3-step and 5-step ahead forecasting proves the effectiveness of the proposed model for future time steps, which is of great importance in the current pandemic situation.

Thus, this research work proposes an effective ANNbased forecasting model, which can be used for forecasting COVID-19 cases, in the state of Karnataka, India showed a gradual increase from May to June 2020 and a steep increase in cases beyond 4 July 2020. To further validate the proposed model, it can be tested using data from the states of India, with increased reported cases similar to Karnataka and may be considering India as a whole. The model has worked well for Hungary data, where the number of reported cases is significantly lower than the state of Karnataka and the increase is very gradual and not as significant as in Karnataka in the last one month. Further, the model can be broad based to include other types of data like number of cases recovered and mortality rate. MLP, a simple, tried and tested ANN model applied in various applications in last 25 years has proved effective for an important application like forecasting the number of infected cases of a pandemic like COVID-19.

\section{Declaration}

Conflict of interest Authors have no conflict of interest to disclose.

\section{References}

1. https://coronavirus.jhu.edu/map.html

2. https://www.worldometers.info/

3. R. Sujath, J.M. Chatterjee, A.E. Hassanien, A machine learning forecasting model for COVID-19 pandemic in India. Stoch. Env. Res. Risk Assess. 34, 959 (2020)

4. G.R. Shinde, A.B. Kalamkar, P.N. Mahalle, N. Dey, J. Chaki, A.E. Hassanien, Forecasting models for coronavirus disease (COVID-19): a survey of the state-of-the-art. SN Comput. Sci. 1(4), 1-15 (2020)

5. H. Swapnarekha, H.S. Behera, J. Nayak, B. Naik, Role of intelligent computing in COVID-19 prognosis: a state-of-the-art review. Chaos Solitons Fractals Non linear Sci. Nonequilib. Complex Phenom. 138, 109947 (2020)

6. G. Pinter, I. Felde, A. Mosavi, P. Ghamisi, R. Gloaguen, COVID19 pandemic prediction for hungary; a hybrid machine learning approach. Mathematics 8(6), 890 (2020)

7. F. Rustam, A.A. Reshi, A. Mehmood, S. Ullah, B.W. On, W. Aslam, G.S. Choi, COVID-19 future forecasting using supervised machine learning models. IEEE Access 8, 101489 (2020). https://doi.org/10.1109/ACCESS.2020.2997311 (Digital Object Identifier)

8. A.I. Saba, A.H. Elsheikh, Forecasting the prevalence of COVID19 outbreak in Egypt using nonlinear autoregressive artificial neural networks. Process Saf. Environ. Prot. 141, 1 (2020)

9. N. Hasan, A methodological approach for predicting COVID-19 epidemic using EEMD-ANN hybrid model. Internet Things 11, 100228 (2020)

10. Z. Car, S.B. Segota, N. Andelic, I. Lorencin, V. Mrzljak, Modeling the spread of COVID-19 infection using a multilayer peceptron. Hindawi Comput. Math. Methods Med. (2020). https://doi.org/10.1155/2020/5714714 (Article ID 5714714)

11. S.K. Tamang, P.D. Singh, B. Datta, Forecasting of Covid-19 cases based on prediction using artificial neural network curve fitting technique. Glob. J. Environ. Sci. Manag. (GJESM) 6(4), 53-64 (2020)

12. A. Mollalo, K.M. Rivera, B. Vahedi, Artificial neural network modeling of novel coronavirus (COVID-19) incidence rates across the Continental United States. Int. J. Environ. Res. Public Health 17, 4204 (2020)

13. S. Haykin, Neural Networks: A Comprehensive Foundation, 2nd edn. (Pearson Education, London, 2009).

14. G.B. Huang, Q.Y. Zhu, C.K. Siew, Extreme learning machine: theory and applications. Neurocomputing 70(1-3), 489-501 (2006)

15. S. Ding, X. Xu, R. Nie, Extreme learning machine and its applications. Neural Comput. Appl. 25(3-4), 549-556 (2014)

16. X.S. Yang, S. Deb, Cuckoo search via Lévy flights. In 2009 world congress on nature \& biologically inspired computing (NaBIC), 210-214 (2009)

17. R.P. Shetty, A. Sathyabhama, P.S. Pai, Wind power optimization: a comparison of meta-heuristic algorithms. MS\&E 376(1), 012021 (2018)

18. R.P. Shetty, A. Sathyabhama, P.S. Pai, An efficient online sequential extreme learning machine model based on feature selection and parameter optimization using cuckoo search algorithm for multi-step wind speed forecasting. Soft Comput. 25(2), 1277-1295 (2021)

19. G.E.P. Box, G.M. Jenkins, G.C. Reinsel, Time Series Analysis, Forecasting and Control, 4th edn. (Wiley, Hoboken, 2008). (ISBN 9780470272848)

20. P. Civicioglu, B. Erkan, A conceptual comparison of the Cuckoosearch, particle swarm optimization, differential evolution and artificial bee colony algorithms. Artif. Intell. Rev. 39(4), 315-346 (2013)

21. S.F. Ardabili, A. Mosavi, P. Ghamisi, F. Ferdinand, A.R. Varkonyi-Koczy, U. Reuter, T. Rabczuk, P.M. Atkinson, Covid-19 outbreak prediction with machine learning. Algorithms 13(10), 249 (2020)

22. T. Shreshth, S. Tuli, R. Tuli, S.S. Gill, Predicting the growth and trend of COVID-19 pandemic using machine learning and cloud computing. Internet Things 11, 100222 (2020)

Publisher's Note Springer Nature remains neutral with regard to jurisdictional claims in published maps and institutional affiliations. 